\title{
Phonon thermal conductivity of scandium nitride for thermoelectrics from rst- principles calculations and thin-Im growth
}

Kerdsongpanya, Sit; Hellman, Olle ; Sun, Bo ; Koh, Yee Kan ; Lu, Jun; Van Nong, Ngo; Simak, Sergei I. ; Alling, Björn ; Eklund, Per

Published in:

Physical Review B

Link to article, DOI:

10.1103/PhysRevB.96.195417

Publication date:

2017

Document Version

Peer reviewed version

Link back to DTU Orbit

Citation (APA):

Kerdsongpanya, S., Hellman, O., Sun, B., Koh, Y. K., Lu, J., Van Nong, N., Simak, S. I., Alling, B., \& Eklund, P. (2017). Phonon thermal conductivity of scandium nitride for thermoelectrics from rst-principles calculations and thin-Im growth. Physical Review B, 96(19), [195417]. https://doi.org/10.1103/PhysRevB.96.195417

\section{General rights}

Copyright and moral rights for the publications made accessible in the public portal are retained by the authors and/or other copyright owners and it is a condition of accessing publications that users recognise and abide by the legal requirements associated with these rights.

- Users may download and print one copy of any publication from the public portal for the purpose of private study or research.

- You may not further distribute the material or use it for any profit-making activity or commercial gain

- You may freely distribute the URL identifying the publication in the public portal 


\title{
Phonon thermal conductivity of scandium nitride for thermoelectrics from first-principles calculations and thin-film growth
}

Sit Kerdsongpanya,,${ }^{1,}$ Olle Hellman, ${ }^{1,2}$ Bo Sun, ${ }^{3}$ Yee Kan Koh, ${ }^{3}$ Jun Lu, ${ }^{1}$ Ngo Van Nong, ${ }^{4}$ Sergei I. Simak, ${ }^{1}$ Björn Alling, ${ }^{1,5}$ and Per Eklund ${ }^{1, \dagger}$

${ }^{1}$ Department of Physics, Chemistry, and Biology (IFM), Linköping University, SE-581 83 Linköping, Sweden

${ }^{2}$ Department of Applied Physics and Materials Science, California Institute of Technology, Pasadena, California 91125, USA

${ }^{3}$ Department of Mechanical Engineering, National University of Singapore, Block EA, 9 Engineering Drive 1, \#07-08, 117576 Singapore

${ }^{4}$ Department of Energy Conversion and Storage, Technical University of Denmark, Ris $\phi$ Campus, Frederiksborgvej 399, Building 779, 4000 Roskilde, Denmark

${ }^{5}$ Max-Planck-Institut für Eisenforschung GmbH, D-40237 Düsseldorf, Germany

(Received 2 July 2016; revised manuscript received 6 October 2017; published xxxxxx)

\begin{abstract}
The knowledge of lattice thermal conductivity of materials under realistic conditions is vitally important since many modern technologies require either high or low thermal conductivity. Here, we propose a theoretical model for determining lattice thermal conductivity, which takes into account the effect of microstructure. It is based on $a b$ initio description that includes the temperature dependence of the interatomic force constants and treats anharmonic lattice vibrations. We choose $\mathrm{ScN}$ as a model system, comparing the computational predictions to the experimental data by time-domain thermoreflectance. Our experimental results show a trend of reduction in lattice thermal conductivity with decreasing domain size predicted by the theoretical model. These results suggest a possibility to control thermal conductivity by microstructural tailoring and provide a predictive tool for the effect of the microstructure on the lattice thermal conductivity of materials based on ab initio calculations.
\end{abstract}

DOI: 10.1103/PhysRevB.00.005400

Design of modern materials inevitably requires taking the thermal conductivity into account [1]. For thermoelectric materials a low thermal conductivity is crucial to avoid heat transfer across the legs and therefore loss in energy conversion efficiency [2]. In contrast, electronic components require packaging materials with high thermal conductivity to efficiently dissipate the generated heat [3]. Furthermore, the more complex the devices become, the higher is the demand to control the interplay between microstructure and thermal performance [4,5]. For hard protective wear-resistant coatings, in, e.g., cutting or milling applications, thermal properties are often barely considered when optimizing mechanical and tribological properties, despite the fact that the workpiece is typically subject to temperatures locally exceeding $1100^{\circ} \mathrm{C}$ in the contact spots [6-8]. The requirements for thermal conductivity are therefore high: the in-plane heat spread within the coating should be as high as possible to ensure uniform heating, while the cross-plane thermal conductivity should be as low as possible to minimize the heat load on the substrate [6-8].These examples underscore how important it is to understand and be able to tailor thermal conductivity in materials in a broad range of applications.

Measurements of thermal conductivity are relatively standard for bulk materials but much more challenging for thin films and nanoscale materials, still being the topic of active method development $[9,10]$. There is therefore a substantial need to also develop theoretical methods for predicting or simulating the thermal conductivity of real materials. Recent advances in methodology allow us to predict the thermal

\footnotetext{
*Present address: Department of Materials Science and Engineering, Rensselaer Polytechnic Institute, Troy, New York 12180, USA.

${ }^{\dagger}$ Corresponding author: per.eklund@liu.se
}

conductivity from first principles. In the present paper, 53 we combine state-of-the-art computational techniques with 54 characterization and measurements to determine the effect of 55 microstructure on the thermal conductivity of ScN thin films. 56

When discussing thermal properties from a computational ${ }_{57}$ perspective, the lattice dynamical Hamiltonian

$$
\begin{aligned}
H= & U_{0}+\sum_{i} \frac{p_{i}^{2}}{2 m_{i}}+\frac{1}{2 !} \sum_{i j \alpha \beta} \Phi_{i j}^{\alpha \beta} u_{i}^{\alpha} u_{j}^{\beta} \\
& +\frac{1}{3 !} \sum_{i j k \alpha \beta \gamma} \Phi_{i j k}^{\alpha \beta \gamma} u_{i}^{\alpha} u_{j}^{\beta} u_{k}^{\gamma}+\ldots,
\end{aligned}
$$

is the starting point. It relates the energy of the lattice 59 to displacements $(u)$ of atoms from equilibrium, with a 60 proportionality constant $(\Phi)$ for each pair, triplet, quartet, and 61 so on, where ijk are indices of atoms and $\alpha \beta \gamma$ are Cartesian 62 indices. Traditionally, these interatomic force constants $\Phi{ }_{63}$ are determined by the Taylor expansion of the zero-Kelvin 64 energy around the equilibrium positions, assuming that this 65 expansion is also valid at high temperatures. In this paper we 66 use a recently developed technique, the temperature-dependent 67 effective potential (TDEP) [11-13], where $\Phi$ 's are extracted 68 from the finite-temperature $a b$ initio molecular dynamics. 69 This provides their intrinsic temperature dependence and the 70 ability to model phenomena inaccessible by traditional means 71 $[14,15]$.

To address these general research questions, we choose 73 scandium nitride as a model system. There is an increasing 74 interest in $\mathrm{ScN}$ for a wide range of applications [16], such 75 as a dislocation-reducing buffer for group III nitrides [17] or 76 as a potential thermoelectric material because of its relatively 77 large Seebeck coefficient $S$ accompanied by low-resistivity 78 $\rho$ that results in a high thermoelectric power factor $\left(S^{2} / \rho\right) 79$ of about $2.5-3.3 \times 10^{-3} \mathrm{~W} \mathrm{~m}^{-1} \mathrm{~K}^{-2}$ [18-21]. ScN can also 80 
${ }_{81}$ be made $p$ type by doping on Sc sites [22,23]. The former 82 application requires a high thermal conductivity, while the 83 latter requires it to be low. With an indirect gap of $0.9 \mathrm{eV}$, 84 the thermal conductivity of $\mathrm{ScN}$ will be dominated by the 85 lattice contribution [24-27]. In an ideal harmonic material, 86 nothing prevents phonons from carrying heat. In practice, 87 scattering from impurities or isotopes, grain boundaries, and 88 the intrinsic anharmonicity result in a finite phonon lifetime. ${ }_{89} \mathrm{Sc}$ is isotopically pure. A large single crystal of pure $\mathrm{ScN}$ 90 would leave the intrinsic anharmonicity as the sole scattering 91 mechanism. Any alloying dopant would decrease thermal 92 conductivity more than in a material with natural isotopic 93 defects, as the majority of heat is carried by acoustic phonons, 94 rendering the $\mathrm{N}$ isotopic distribution less important. This is 95 therefore an opportunity for tailoring the thermal conductivity, 96 by controlling the microstructure.

The temperature-dependent phonon thermal conductivity of $\mathrm{ScN}$ was obtained with ab initio Born-Oppenheimer molecular 99 dynamics (AIMD). All calculations in this work were carried 100 out using the projector augmented wave method [28] as 01 implemented in the Vienna Ab initio Simulation Package 102 (VASP) [29-32]. The AIMD temperature was set to 400, 800, 1031200 , and $1600 \mathrm{~K}$ and the volumes to $83.74,88.12,92.65$, 10497.34 , and $103.82 \AA^{3}$ per unit cell for 20 calculations in 105 total, where each calculation was run for 20000 time steps 106 of 1 fs each with a $5 \times 5 \times 5$ supercell ( 250 atoms in total). 107 Temperature was controlled with a Nosé-Hoover thermostat $108[33,34]$. The exchange correlation was approximated with the 109 AM05 approximation [35,36] and we used the $\Gamma$ point for 110 the Brillouin-zone integration along with a plane-wave cutoff 111 of $400 \mathrm{eV}$. The AIMD data were postprocessed using the 1112 TDEP [11-13] and the free Gibbs free-energy surface was 113 constructed. From that we extracted volume as a function of 114 temperature, and interpolated the interatomic force constants 115 to the volumes corresponding to zero pressure at the respective 116 temperatures. At these volumes we used the full solution 117 to the linearized phonon Boltzmann equation [37,38] and 118 calculated the lattice contribution to the thermal conductivity 119 on a $31 \times 31 \times 31 q$-point grid.

$120 \mathrm{ScN}$ thin films were grown on one-side-polished sapphire $121 \mathrm{Al}_{2} \mathrm{O}_{3}(0001)$ (dimensions $10 \times 10 \times 0.5 \mathrm{~mm}^{3}$ ) by reactive $122 \mathrm{dc}$ magnetron sputtering in a high-vacuum chamber with a 123 base pressure of $2.7 \times 10^{-5} \mathrm{~Pa}$. The system is described in 124 detail elsewhere [39]. The Sc target was a 7.62-cm-diameter 125 disk shape, with $99.95 \%$ purity with fluorine as a common 126 impurity. Prior to deposition, the substrates were degreased 127 in an ultrasonic bath with trichloroethylene, acetone, and 128 isopropanol for $5 \mathrm{~min}$ each and subsequently blown dry with ${ }_{129} \mathrm{~N}_{2}$. The Sc target was operated in dc mode (power regulated) at 130 a power of $120 \mathrm{~W}$. The substrate was rotated during deposition 131 in order to obtain uniform films. A substrate bias of $-30 \mathrm{~V}$ bias 132 was applied to the substrate during deposition. The depositions 133 were performed in $\mathrm{Ar} / \mathrm{N}_{2}$ atmosphere at the total gas pressure 134 of $0.67 \mathrm{~Pa}$ with $0.27 \mathrm{~Pa} \mathrm{Ar}$ and $0.4 \mathrm{~Pa} \mathrm{~N}_{2}$. Before deposition, the 135 substrates were heated in vacuum to the deposition temperature ${ }_{136}$ (for $1 \mathrm{~h}$ for temperature stabilization and degassing). The ${ }_{137}$ deposition temperatures $\left(T_{\mathrm{d}}\right)$ were $400,500,600$, and $700{ }^{\circ} \mathrm{C}$. ${ }_{138}$ The crystal structure and average characteristic $\mathrm{x}$-ray 139 scattering domain sizes of as-deposited films were determined by x-ray diffraction (XRD) with standard $\theta-2 \theta$ scans using 140 $\mathrm{CuK} \alpha$ radiation in a Philips PW 1820 diffractometer. Cross- ${ }_{141}$ section transmission electron microscopy (TEM) and plan- 142 view scanning electron microscopy (SEM) were performed on 143 all samples to investigate film microstructure and morphology. 144 For cross-section TEM, the samples were prepared by gluing 145 two pieces of the sample face to face into a Ti grid, ${ }_{146}$ then polishing down to 50- $\mu \mathrm{m}$ thickness. Ion milling was 147 performed in a Gatan Precision Ion Polishing System at 148 $\mathrm{Ar}^{+}$energy of $5 \mathrm{keV}$ and a gun angle of $5^{\circ}$, with a final ${ }_{149}$ polishing step with 2-keV $\mathrm{Ar}^{+}$energy and angle of $2^{\circ}$. TEM ${ }_{150}$ characterization was performed using a Tecnai G2 TF20UT ${ }_{151}$ with a field-emission gun. A ULVAC-RIKO ZEM3 system 152 was used to measure the Seebeck coefficient and electrical ${ }_{153}$ resistivity of the films simultaneously in a low-pressure helium 154 atmosphere from room temperature. The error bar of these 155 measurements is within 7\%, and the substrate contribution to 156 the Seebeck coefficient and electrical resistivity is negligible. ${ }_{157}$

Thermal conductivities of $\mathrm{ScN}$ samples were measured ${ }_{158}$ using time-domain thermoreflectance (TDTR). We only briefly 159 describe the measurements here; details of the TDTR setup and ${ }_{160}$ implementation can be found in Ref. [40]. Before conducting 161 TDTR measurements, the samples were coated with an $\sim 80-\quad 162$ nm-thick $\mathrm{Al}$ as transducer. In TDTR measurements, laser ${ }_{163}$ pulses from an ultrafast laser are split into a pump beam 164 and a probe beam. The pump beam is modulated and heats 165 the sample periodically, creating a temperature oscillation 166 in the sample. The probe beam monitors the temperature ${ }_{167}$ oscillation at sample surface via thermoreflectance (i.e., 168 change of reflectance with temperature). Since the induced 169 temperature oscillation depends on the thermal properties of 170 the sample, TDTR is an accurate approach to measure the 171 thermal conductance of interface and thermal conductivity of 172 nanostructures [41-43]. In the measurements of ScN samples, 173 we use a $1 / e^{2}$ laser radius of $10 \mu \mathrm{m}$, a modulation frequency of 174 $10 \mathrm{MHz}$, and total laser power of $\sim 40 \mathrm{~mW}$ to limit the steady- 175 state temperature rise to $<10 \mathrm{~K}$. The thermal conductivities 176 of $\mathrm{ScN}$ and thermal conductance of $\mathrm{Al} / \mathrm{ScN}$ are then derived 177 by comparing the TDTR measurements to the calculations of 178 the thermal model [44]. In the analysis, the thickness of Al 179 transducer is determined by picosecond acoustics [45] while ${ }_{180}$ the heat capacity of $\mathrm{ScN}$ is from the literature value of bulk 181 $\mathrm{ScN}$ [46]. The measurement uncertainties are estimated to be 182 $\sim 6 \%$ for all measured thermal conductivities.

Figure 1 shows the calculated dynamical structure factor 184 $S(q, E)$ of $\mathrm{ScN}$ at room temperature, showing signs of strongly 185 anharmonic behavior. There is a faint satellite peak from the ${ }_{186}$ transverse optical (TO) mode around the $\Gamma$ point, owing to the ${ }_{187}$ many available scattering channels between the acoustic and 188 optical branches in this region. This is reminiscent of $\mathrm{PbTe}$ and 189 SnTe [15]. While the zone center shows deviations from the 190 assumption that the line shape is Lorentzian (see Supplemental 191 Material [47]), the TO phonons carry little heat and we can 192 therefore assume that the phonon Boltzmann equation is still 193 valid.

At room temperature, using the Boltzmann transport equa- ${ }_{195}$ tion for phonons, we predict the lattice contribution to the 196 thermal conductivity to be $20 \mathrm{Wm}^{-1} \mathrm{~K}^{-1}$. This is assuming 197 an infinite perfect crystal. This should be compared to 198 previously reported experimental values of $8-12 \mathrm{Wm}^{-1} \mathrm{~K}^{-1}{ }_{199}$ 


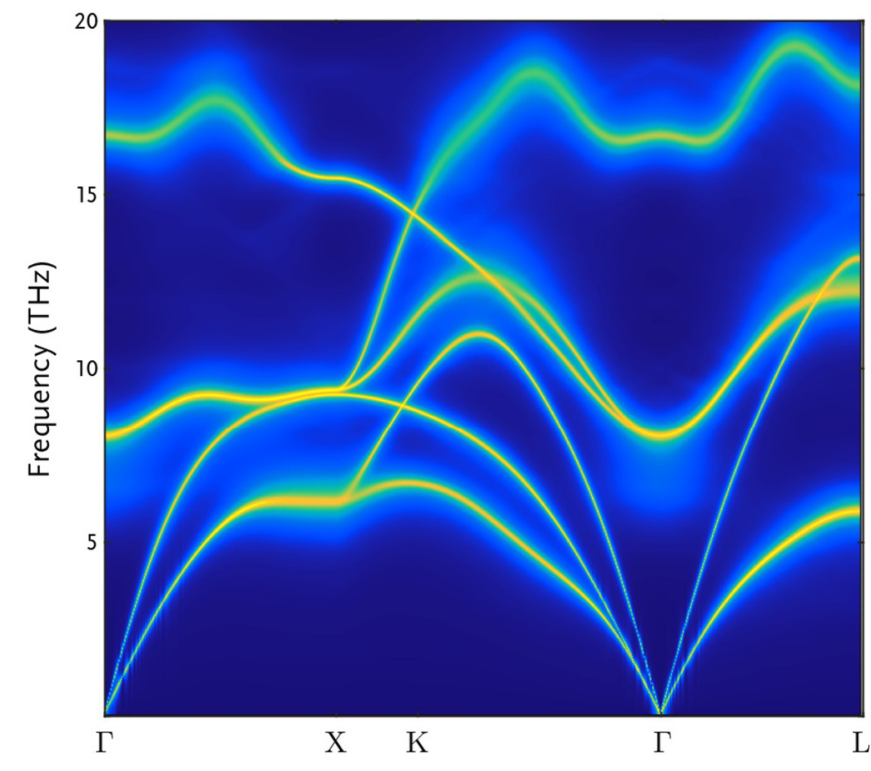

FIG. 1. Dynamical structure factor $S(q, E)$ for $\mathrm{ScN}$ at room temperature from first-principles calculations.

$200[19,48]$. The reason for these differences is nonideal effects 201 of microstructure (and impurities), i.e., that real $\mathrm{ScN}$ is not a 202 perfect crystal. The present synthesis of $\mathrm{ScN}$ thin films allows 203 a systematic, and if not quantitative at least semiquantitative, 204 correlation between microstructure and other nonideal effects 205 on thermal conductivity and theoretical calculations of the 206 same effects.

207 To simulate the microstructural effects, we modify the 208 expression used for the thermal conductivity from

$$
\kappa_{\alpha \alpha}=\frac{1}{V} \sum_{\mathbf{q} s} C_{\mathbf{q} s} v_{\alpha \mathbf{q} s}^{2} \tau_{\alpha \mathbf{q} s},
$$

209 where $V$ is the volume, $v_{\alpha \mathbf{q} s}$ is the mode phonon velocity, $\tau_{\alpha \mathbf{q} s}$ 210 is the mode phonon lifetime corresponding to wave vector $\boldsymbol{q}$ 211 in branch $s$ (with $v_{\alpha \mathbf{q} s}$ and $\tau_{\alpha \mathbf{q} s}$ determined by an iterative 212 solution to the phonon Boltzmann equation [33,34] and $\alpha$ 213 the Cartesian index), and $C_{\mathrm{q} s}=\hbar \omega_{\mathrm{q} s} \partial n_{\mathrm{q} s}^{0} / \partial T$ is the mode 214 specific heat, where $\hbar$ is the reduced Planck's constant $(h / 2 \pi)$, $215 \omega_{\mathbf{q} s}$ is the phonon frequency, and $n_{\mathbf{q} s}^{0}$ is the Bose-Einstein 216 distribution function. By adding the Heaviside step function $217[\Theta(l)]$ as a function of mean scattering domain size $l$, the 218 thermal conductivity including the domain-size effect is

$$
\kappa_{\alpha \alpha}^{\mathrm{acc}}(l)=\frac{1}{V} \sum_{\mathbf{q} s} C_{\mathbf{q} s} v_{\alpha \mathbf{q} s}^{2} \tau_{\alpha \mathbf{q} s} \Theta\left(l-\left|v_{\mathbf{q} s}\right| \tau_{\alpha \mathbf{q} s}\right) .
$$

219

220

This means that to simulate a certain mean domain size, we only include those phonons whose mean-free path is small enough to fit inside the domain. The results can be seen in Fig. 2, together with the experimental values. The model here differs from other approaches that model interfaces [49-51], phenomenologically compute the phonon lifetime [52], or directly include boundary scattering in the Boltzmann transport equation [53].

XRD results and electron microscopy images of all samples (Figs. 3 and 4) show highly textured polycrystalline $\mathrm{ScN}$ films where films were grown in the [111] direction on $\mathrm{Al}_{2} \mathrm{O}_{3}(0001)$.

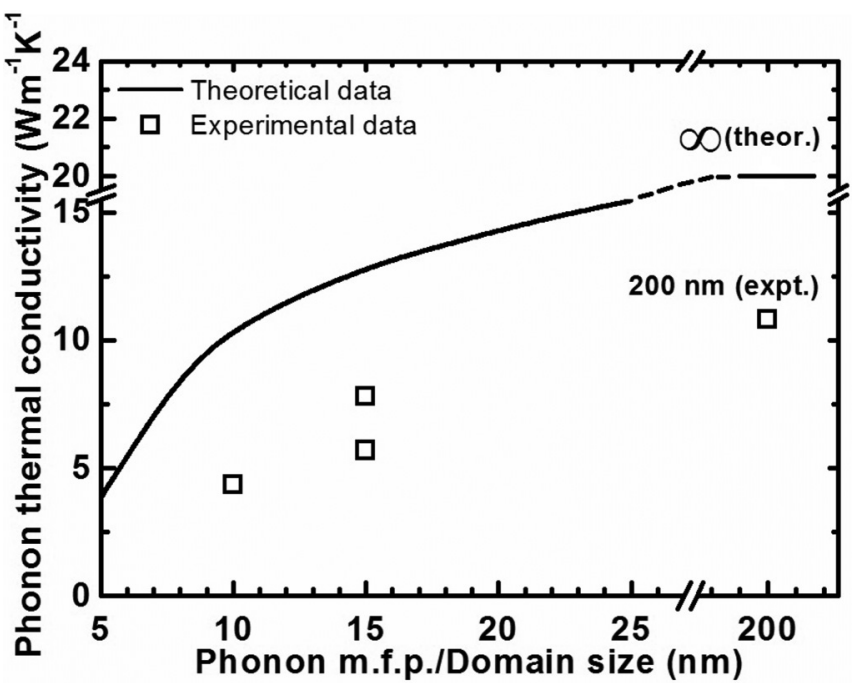

FIG. 2. Theoretical and experimental values of the phonon thermal conductivity of $\mathrm{ScN}$ (assuming defect-free crystal) as a function of phonon mean-free path or characteristic x-ray scattering domain size.

In addition, the sample grown at deposition temperature $\left(T_{\mathrm{d}}\right)_{230}$ of $700^{\circ} \mathrm{C}$ exhibits epitaxial growth, which can be seen from ${ }^{231}$ TEM [Fig. 4(f) and Supplemental Material [47]). Samples 232 grown at $T_{\mathrm{d}}=400-600^{\circ} \mathrm{C}$ show columnar polycrystalline ${ }_{23}$ structure and triangular contrast at the interface between film 234
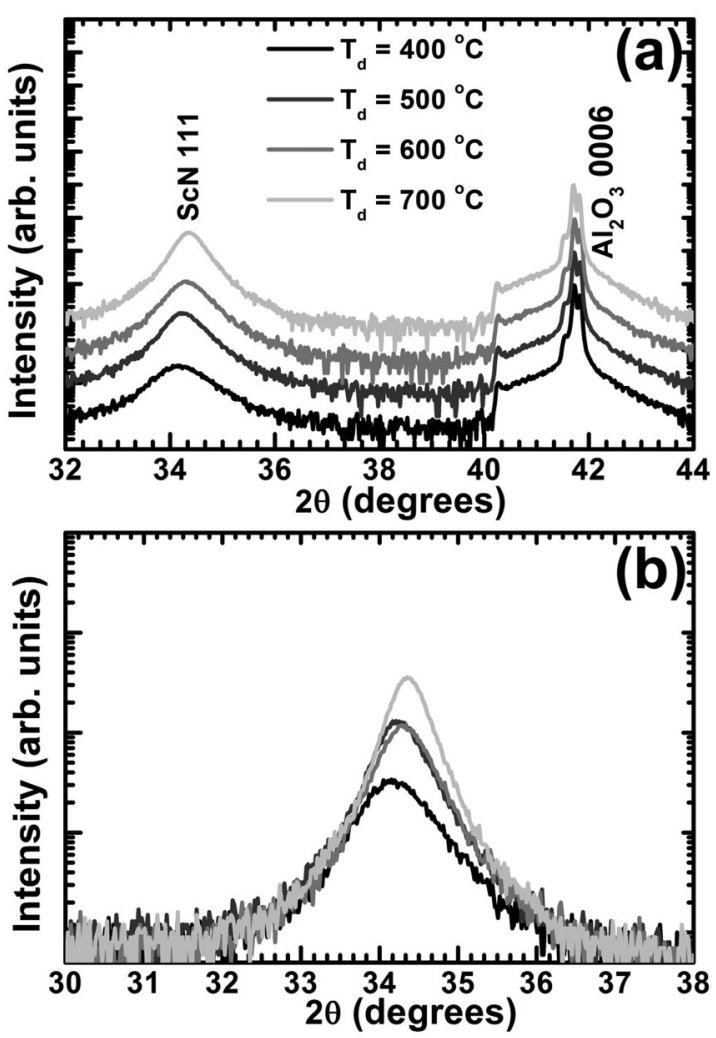

FIG. 3. XRD patterns of $\mathrm{ScN}$ films deposited at deposition temperature of $T_{\mathrm{d}}=400,500,600$, and $700^{\circ} \mathrm{C}$ on $\mathrm{Al}_{2} \mathrm{O}_{3}$. (a) Overview and (b) magnified area around the $\mathrm{ScN} 111$ peak. 


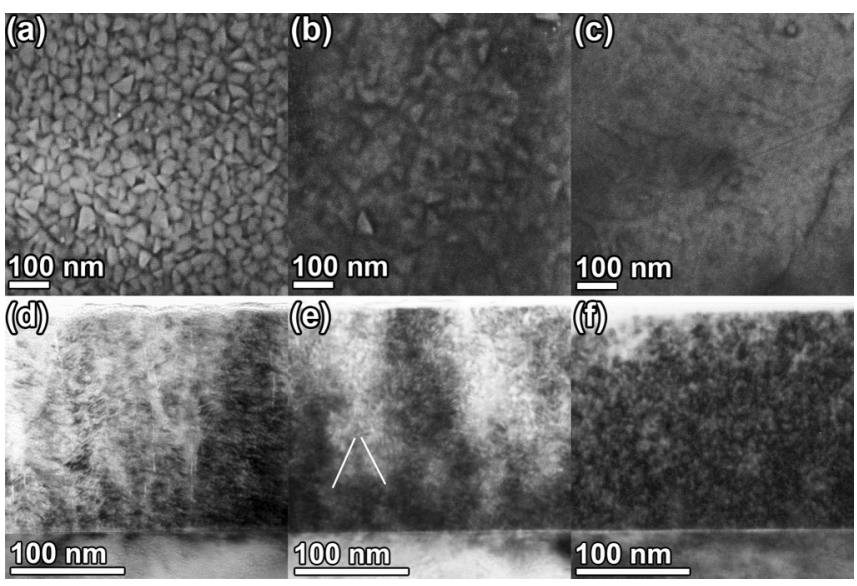

FIG. 4. Electron micrographs of ScN films where (a)-(c) are SEM images and (d)-(f) are cross-section TEM images of the samples that were deposited at deposition temperature, $T_{\mathrm{d}}=400,600$, and $700^{\circ} \mathrm{C}$, respectively.

235 and substrate [Figs. 4(d) and 4(e)]. For more information about 236 samples microstructures see Supplemental Material [47].

From this microstructural information, we see that there 238 are three different types of $\mathrm{ScN}$ samples in this study: (1) 239 sample with small domain size, (2) two samples with essen240 tially the same domain size, and (3) epitaxial thin films, where 241 the domain size is the film thickness. For the polycrystalline 242 films, we can use the characteristic x-ray scattering length 243 in $\theta-2 \theta$ geometry (corresponding to the peak broadening) in 244 order to estimate the domain size of the film in the out-of-plane 245 direction. The characteristic x-ray scattering length is defined 246 as the size of the (out-of-plane) coherently diffracting domains.

247 Therefore, we can estimate the average out-of-plane domain

248 size using Scherrer's formula [54]. From the XRD results, the $2492 \theta$ full width at half maximum of the $\mathrm{ScN} 111$ peaks that 250 are determined by fitting the curve with Lorentz distribution

251 function are $0.80,0.54$, and $0.56^{\circ}$ for the polycrystalline films 252 deposited at 400,500 , and $600^{\circ} \mathrm{C}$, respectively. Thus, the 253 estimates of the average out-of-plane domain size of these $\mathrm{ScN}$ 254 films are 10,15 , and $15 \mathrm{~nm}$ for $T_{\mathrm{d}}=400,500$, and $600{ }^{\circ} \mathrm{C}$, 255 respectively. This is consistent with transmission electron 256 microscopy (see Fig. S4 in Supplemental Material [47]).

All ScN films were grown to the same thickness of 8 about $200 \mathrm{~nm}$ (see Fig. S2 in Supplemental Material [47]). 259 The phonon thermal conductivity of all the samples was so determined from $\kappa_{\mathrm{ph}}=\kappa_{\text {tot }}-\kappa_{\mathrm{e}}$, where $\kappa_{\text {tot }}$ is the total thermal 261 conductivity and $\kappa_{\mathrm{e}}$ is the electronic thermal conductivity, 262 which is described as $\kappa_{\mathrm{e}}=L T / \rho$ where $L$ is the Lorenz 263 number $\left(2.44 \times 10^{-8} \mathrm{~W} \Omega \mathrm{K}^{-2}\right), \rho$ is the electrical resistivity, 264 and $T$ is the absolute temperature. The electrical resistivity of 265 the polycrystalline $\mathrm{ScN}$ films at room temperature is $61.0,11.0$, 266 and $9.8 \mu \Omega \mathrm{m}$ for the films deposited at 400,500 , and $600{ }^{\circ} \mathrm{C}$, 267 respectively. For the epitaxial film, the electrical resistivity 268 is $4.0 \mu \Omega \mathrm{m}$. From the $\kappa_{\mathrm{ph}}=\kappa_{\mathrm{tot}}-\kappa_{\mathrm{e}}$ equation, we can 269 calculate the phonon thermal conductivity from the measured 270 total thermal conductivity at room temperature for all $\mathrm{ScN}$ 271 films. The results are shown in Fig. 2. For polycrystalline 272 films, the phonon thermal conductivity of the samples with 273 domain sizes of 10 and $15 \mathrm{~nm}$ (two samples) are 5.4, 9.7, and $7.1 \mathrm{~W} \mathrm{~m}^{-1} \mathrm{~K}^{-1}$, respectively. The origin of the slight ${ }_{274}$ difference in phonon thermal conductivity between the two 275 15-nm-domain-size samples is likely correlated with the fact 276 that the thermal conductance of the $\mathrm{Al} / \mathrm{ScN}$ interface of 277 the $600{ }^{\circ} \mathrm{C}$ sample is also particularly low (see Fig. S3 in 278 Supplemental Material [47]). This indicates that the quality 279 of the sample (in terms of surface oxidation and/or impurity 280 content) is not as good compared to other samples. On the other ${ }_{281}$ hand, the epitaxial film exhibits a phonon thermal conductivity 282 of $10.5 \mathrm{Wm}^{-1} \mathrm{~K}^{-1}$ which is similar to previous values reported 283 for epitaxial $\mathrm{ScN}$ films $[19,55]$.

Overall, the results show an increase of phonon thermal 285 conductivity with increasing domain size. These results agree 286 well with our calculated phonon thermal conductivity, but with 287 a systematic difference of a factor of $\sim 2$. For example, the 288 predicted phonon thermal conductivity of a polycrystalline 289 film with 10 -nm domain size is $\sim 10.3 \mathrm{Wm}^{-1} \mathrm{~K}^{-1}$, while the 290 experimental value is $\sim 5.4 \mathrm{Wm}^{-1} \mathrm{~K}^{-1}$. The reason for this 291 systematic shift is additional effects, other than domain-size 292 effect, that are not accounted for in the model. Including film 293 thickness and impurity scattering reduces the calculated values 294 close to the experimental ones. Oxygen impurities on N sites 295 (known in $\mathrm{ScN}$ films) require high concentrations to affect the 296 thermal conductivity. A concentration of 5 at. \% oxygen only ${ }_{297}$ reduces thermal conductivity by at most $1 \%$ at $300 \mathrm{~K}$. However, 298 since $\mathrm{Sc}$ is isotopically pure, any impurities on Sc sites will 299 have a substantial effect on thermal conductivity. Introduction 300 of a fraction of a percent of vacancies, antisite, or substitutional 301 defects (data not shown, as we do not have experimental 302 information on the concentration of Sc-site defects) decreases 303 the phonon lifetimes for the Sc-dominated acoustic branches 304 substantially, lowering the lattice thermal conductivity by as 305 much as $50 \%$. These results and observations validate the 306 model for calculating effects of microstructure on thermal 307 conductivity and enable comparisons of measurements of ther- 308 mal conductivity of real materials with calculations separating 309 different effects.

In conclusion, we have theoretically and experimentally 311 investigated phonon thermal conductivity in $\mathrm{ScN}$ thin films. 312 Using a theoretical model accounting for microstructural 313 effects on the thermal conductivity, we can semiquantitatively 314 predict the thermal conductivity of $\mathrm{ScN}$. The predictions sys- 315 tematically overestimate the thermal conductivity by a factor 316 of $\sim 2$, which is due to additional effects, including impurity 317 scattering, which can in principle also be incorporated into the 318 model to improve the quantitative agreement. The dominant 319 effect is domain-size reduction of the thermal conductivity as 320 evidenced by the calculated values of $20 \mathrm{Wm}^{-1} \mathrm{~K}^{-1}$ for an ${ }_{321}$ ideal single crystal and $4-13 \mathrm{Wm}^{-1} \mathrm{~K}^{-1}$ for domain sizes of 322 5-20 nm. This large reduction in thermal conductivity with ${ }_{323}$ grain size agrees well with our experiments on $\mathrm{ScN}$, and 324 more generally offers an approach for integrated theoretical- 325 experimental design of the thermal conductivity of real 326 materials.

The research leading to these results has received fund- 328 ing from the European Research Council under the Euro- ${ }_{329}$ pean Community's Seventh Framework Programme (Grant ${ }_{330}$ No. FP/2007-2013)/ERC Grant Agreement No. 335383, the 331 
332 Swedish Government Strategic Research Area in Materials ззз Science on Functional Materials at Linköping University 334 (Faculty Grant SFO-Mat-LiU No. 2009 00971), the Swedish 335 Research Council (VR) under Projects No. 2012-4430 and No. 336 2016-03365 (S.K. and P.E.), No. 330-2014-6336 (B.A.), No. 337 2014-4750 (S.I.S.), No. 637-2013-7296 (O.H.), the Linnaeus 338 Environment LiLi-NFM, and the Swedish Foundation for 339 Strategic Research (SSF) through the Future Research Leaders
5 Program. The calculations were performed using computer 340 resources provided by the Swedish National Infrastructure ${ }_{341}$ for Computing (SNIC) at the National Supercomputer Centre 342 (NSC). N.V.N. would like to acknowledge the financial support 343 by the NanoCaTe project (FP7-NMP No. 604647). B.S. and 344 Y.K.K. acknowledge the National University of Singapore 345 Startup Grant.

S.K. and O.H. contributed equally to this work.
[1] E. S. Toberer, L. L. Baranowski, and C. Dames, Advances in thermal conductivity, Annu. Rev. Mater. Res. 42, 179 (2012).

[2] G. J. Snyder and E. S. Toberer, Complex thermoelectric materials, Nat. Mater. 7, 105 (2008).

[3] X. Liu et al., Thermal management strategies for high power semiconductor pump lasers, IEEE Trans. Compon. Packag. Technol. 29, 268 (2006).

[4] R. Cheaito, J. C. Duda, T. E. Beechem, K. Hattar, J. F. Ihlefeld, D. L. Medlin, M. A. Rodriguez, M. J. Campion, E. S. Piekos, and P. E. Hopkins, Experimental Investigation of Size Effects on the Thermal Conductivity of Silicon-Germanium Alloy Thin Films, Phys. Rev. Lett. 109, 195901 (2012).

[5] C. J. Vineis, A. Shakouri, A. Majumdar, and M. G. Kanatzidis, Nanostructured thermoelectrics: Big efficiency gains from small features, Adv. Mater. 22, 3970 (2010).

[6] P. H. M. Bottger et al., Thermal conductivity of hard oxynitride coatings, Thin Solid Films 549, 232 (2013).

[7] P. H. M. Böttger et al., Anisotropic layered media with microinclusions: Thermal properties of arc-evaporation multilayer metal nitrides, Int. J. Therm. Sci. 77, 75 (2014).

[8] P. H. M. Böttger et al., Hard wear-resistant coatings with anisotropic thermal conductivity for high thermal load applications, J. Appl. Phys. 116, 013507 (2014).

[9] D. G. Cahill et al., Nanoscale thermal transport, J. Appl. Phys. 93, 793 (2003).

[10] D. G. Cahill et al., Nanoscale thermal transport. II. 2003-2012, Appl. Phys. Rev. 1, 011305 (2014).

[11] O. Hellman, I. A. Abrikosov, and S. I. Simak, Lattice dynamics of anharmonic solids from first principles, Phys. Rev. B 84, 180301 (2011).

[12] O. Hellman and I. A. Abrikosov, Temperature-dependent effective third-order interatomic force constants from first principles, Phys. Rev. B 88, 144301 (2013).

[13] O. Hellman, P. Steneteg, I. A. Abrikosov, and S. I. Simak, Temperature dependent effective potential method for accurate free energy calculations of solids, Phys. Rev. B 87, 104111 (2013).

[14] J. D. Budai et al., Metallization of vanadium dioxide driven by large phonon entropy, Nature (London) 515, 535 (2014).

[15] C. W. Li et al., Phonon Self-Energy and Origin of Anomalous Neutron Scattering Spectra in SnTe and PbTe Thermoelectrics, Phys. Rev. Lett. 112, 175501 (2014).

[16] S. W. King, R. F. Davis, and R. J. Nemanich, Gas source molecular beam epitaxy of scandium nitride on silicon carbide and gallium nitride surfaces, J. Vac. Sci. Technol. A 32, 061504 (2014).
[17] M. J. Kappers et al., Interlayer methods for reducing the dislocation density in gallium nitride, Physica B 401-402, 296 (2007).

[18] S. Kerdsongpanya et al., Anomalously high thermoelectric power factor in epitaxial $\mathrm{ScN}$ thin films, Appl. Phys. Lett. 99, 232113 (2011).

[19] P. V. Burmistrova et al., Thermoelectric properties of epitaxial $\mathrm{ScN}$ films deposited by reactive magnetron sputtering onto MgO(001) substrates, J. Appl. Phys. 113, 153704 (2013).

[20] P. Eklund, S. Kerdsongpanya, and B. Alling, Transition-metalnitride-based thin films as novel energy harvesting materials, J. Mater. Chem. C 4, 3905 (2016).

[21] P. V. Burmistrova, D. N. Zakharov, T. Favaloro, A. Mohammed, E. A. Stach, A. Shakouri, and T. D. Sands, Effect of deposition pressure on the microstructure and thermoelectric properties of epitaxial $\mathrm{ScN}(001)$ thin films sputtered onto $\mathrm{MgO}(001)$ substrates, J. Mater. Res. 30, 626 (2015).

[22] B. Saha, M. Garbrecht, J. A. Perez-Taborda, M. H. Fawey, Y. R. Koh, A. Shakouri, M. Martin-Gonzalez, L. Hultman, and T. D. Sands, Compensation of native donor doping in ScN: Carrier concentration control and $p$-type ScN, Appl. Phys. Lett. 110, 252104 (2017).

[23] B. Saha, G. Naik, V. Drachev, A. Boltasseva, E. E. Marinero, and T. D. SandsElectronic and optical properties of $\mathrm{ScN}$ and $(\mathrm{Sc}, \mathrm{Mn}) \mathrm{N}$ thin films deposited by reactive DC-magnetron sputtering, J. Appl. Phys. 114, 063519 (2013).

[24] H. A. H. Al-Brithen, A. R. Smith, and D. Gall, Surface and bulk electronic structure of $\mathrm{ScN}(001)$ investigated by scanning tunneling microscopy/spectroscopy and optical absorption spectroscopy, Phys. Rev. B 70, 045303 (2004).

[25] S. Kerdsongpanya, B. Alling, and P. Eklund, Effect of point defects on the electronic density of states of $\mathrm{ScN}$ studied by first-principles calculations and implications for thermoelectric properties, Phys. Rev. B 86, 195140 (2012).

[26] S. Kerdsongpanya, B. Alling, and P. Eklund, Phase stability of ScN-based solid solutions for thermoelectric applications from first-principles calculations, J. Appl. Phys. 114, 073512 (2013).

[27] C. Li and D. Broido, Phonon thermal transport in transitionmetal and rare-earth nitride semiconductors from first principles, Phys. Rev. B 95, 205203 (2017).

[28] P. E. Blöchl, Projector augmented-wave method, Phys. Rev. B 50, 17953 (1994).

[29] G. Kresse and J. Hafner, Ab initio molecular dynamics for openshell transition metals, Phys. Rev. B 48, 13115 (1993).

[30] G. Kresse and D. Joubert, From ultrasoft pseudopotentials to the projector augmented-wave method, Phys. Rev. B 59, 1758 (1999). 
[31] G. Kresse and J. Furthmüller, Efficient iterative schemes for ab initio total-energy calculations using a plane-wave basis set, Phys. Rev. B 54, 11169 (1996).

[32] G. Kresse and J. Furthmüller, Efficiency of ab-initio total energy calculations for metals and semiconductors using a plane-wave basis set, Comput. Mater. Sci. 6, 15 (1996).

[33] S. Nosé, A molecular dynamics method for simulations in the canonical ensemble, Mol. Phys. 52, 255 (1984).

[34] W. G. Hoover, Canonical dynamics: Equilibrium phase-space distributions, Phys. Rev. A 31, 1695 (1985).

[35] R. Armiento and A. E. Mattsson, Functional designed to include surface effects in self-consistent density functional theory, Phys. Rev. B 72, 085108 (2005).

[36] A. E. Mattsson and R. Armiento, Implementing and testing the AM05 spin density functional, Phys. Rev. B 79, 155101 (2009).

[37] A. Ward, D. A. Broido, D. A. Stewart, and G. Deinzer, Ab initio theory of the lattice thermal conductivity in diamond, Phys. Rev. B 80, 125203 (2009).

[38] M. Omini and A. Sparavigna, An iterative approach to the phonon Boltzmann equation in the theory of thermal conductivity, Physica B 212, 101 (1995).

[39] F. Eriksson, G. A. Johansson, H. M. Hertz, and J. Birch, Enhanced soft x-ray reflectivity of $\mathrm{Cr} / \mathrm{Sc}$ multilayers by ionassisted sputter deposition, Opt. Eng. 41, 2903 (2002).

[40] B. Sun and Y. K. Koh, Understanding and eliminating artifact signals from diffusely scattered pump beam in measurements of rough samples by time-domain thermoreflectance (TDTR), Rev. Sci. Instrum. 87, 064901 (2016).

[41] O. Cometto et al., Vertically self-ordered orientation of nanocrystalline hexagonal boron nitride thin films for enhanced thermal characteristics, Nanoscale 7, 18984 (2015).

[42] J. Ju et al., Thermoelectric properties of In-rich InGaN and InN/InGaN superlattices, AIP Adv. 6, 045216 (2016).

[43] B. Huang and Y. K. Koh, Improved topological conformity enhances heat conduction across metal contacts on transferred graphene, Carbon 105, 268 (2016).
[44] D. G. Cahill, Analysis of heat flow in layered structures for timedomain thermoreflectance, Rev. Sci. Instrum. 75, 5119 (2004).

[45] C. Thomsen, H. T. Grahn, H. J. Maris, and J. Tauc, Surface generation and detection of phonons by picosecond light pulses, Phys. Rev. B 34, 4129 (1986).

[46] B. Saha, J. Acharya, T. D. Sands, and U. V. Waghmare, Electronic structure, phonons, and thermal properties of $\mathrm{ScN}$, $\mathrm{ZrN}$, and HfN: A first-principles study, J. Appl. Phys. 107, 033715 (2010).

[47] See Supplemental Material at http://link.aps.org/supplemental/ 10.1103/PhysRevB.xx.xxxxxx for (brief description).

[48] V. Rawat, D. N. Zakharov, E. A. Stach, and T. D. Sands, Pseudomorphic stabilization of rocksalt $\mathrm{GaN}$ in TiN/GaN multilayers and superlattices, Phys. Rev. B 80, 024114 (2009).

[49] H.-S. Kim, S. D. Kang, Y. Tang, R. Hanus and G. J. Snyder, Dislocation strain as the mechanism of phonon scattering at grain boundaries, Mater. Horiz. 3, 234 (2016).

[50] P. Hołuj, C. Euler, B. Balke, U. Kolb, G. Fiedler, M. M. Müller, T. Jaeger, E. Chávez Angel, P. Kratzer, and G. Jakob, Reduced thermal conductivity of TiNiSn/HfNiSn superlattices, Phys. Rev. B 92, 125436 (2015).

[51] P. Chen, N. A. Katcho, J. P. Feser, W. Li, M. Glaser, O. G. Schmidt, D. G. Cahill, N. Mingo, and A. Rastelli, Role of Surface-Segregation-Driven Intermixing on the Thermal Transport through Planar Si/Ge Superlattices, Phys. Rev. Lett. 111, 115901 (2013).

[52] N. Mingo and D. A. Broido, Length dependence of carbon nanotube thermal conductivity and the problem of long waves, Nano Lett. 5, 1221 (2005).

[53] G. Fugallo, M. Lazzeri, L. Paulatto, and F. Mauri, Ab initio variational approach for evaluating lattice thermal conductivity, Phys. Rev. B 88, 045430 (2013).

[54] M. Birkholz, Thin Film Analysis by X-Ray Scattering (WileyVCH, Weinheim, 2006).

[55] V. Rawat, Y. K. Koh, D. G. Cahill, and T. D. Sands, Thermal conductivity of (Zr,W)N/ScN, J. Appl. Phys. 105, 024909 (2009). 